\title{
Long non-coding RNA XIST predicting advanced clinical parameters in cancer: A Meta-Analysis and case series study in a single institution (Review)
}

\author{
CHANGKAI DENG ${ }^{1 *}$, XIANLIANG HU ${ }^{1 *}, \mathrm{KE} \mathrm{WU}^{2}, \mathrm{JUAN} \mathrm{TAN}^{3}$ and CHAO YANG ${ }^{4,5}$ \\ ${ }^{1}$ Department of Pediatric Surgery, Chengdu Women's and Children's Central Hospital of Chongqing Medical University, \\ Chengdu, Sichuan 610069; ${ }^{2}$ Department of Pharmacology, Chongqing Key Laboratory of Biochemistry and \\ Molecular Pharmacology, Chongqing Medical University, Chongqing 400014; ${ }^{3}$ Outpatient Department, \\ The Children's Hospital of Chongqing Medical University, Chongqing 401122; Departments of ${ }^{4}$ Pediatric \\ Surgical Oncology and ${ }^{5}$ Ministry of Education Key Laboratory of Child Development and Disorders, \\ The Children's Hospital of Chongqing Medical University, Chongqing 400014, P.R. China
}

Received August 12, 2018; Accepted June 13, 2019

DOI: $10.3892 / \mathrm{ol} .2019 .10592$

\begin{abstract}
Dysregulated expression of long non-coding RNA $\mathrm{X}$-inactive specific transcript (IncRNA-XIST) has been indicated in various cancer types. In the present study, a meta-analysis was conducted to evaluate the potential role of lncRNA-XIST in predicting the clinicopathological parameters of patients with cancer. Eligible studies were obtained through a systematic search of PubMed, Web of Science, Embase and the Cochrane Library, of articles published prior to January 2019. The combined odds ratio and 95\% confidence interval were calculated to determine the association between lncRNA-XIST expression and patient outcome. In addition, 45 pairs of osteosarcoma (OS) tissues and adjacent healthy tissues from a single institution were analyzed for the expression of IncRNA-XIST, and its association with clinicopathological features; ultimately, a total of 1,869 cancer patients from 25 studies were assessed. The results demonstrated that high expression levels of lncRNA-XIST were significantly associated with lymphatic metastasis, larger tumor size, advanced cancer stage and distant metastasis.
\end{abstract}

Correspondence to: Dr Chao Yang, Department of Pediatric Surgical Oncology, The Children's Hospital of Chongqing Medical University, 136 Zhongshan 2nd Road, Yuzhong, Chongqing 400014, P.R. China

E-mail: 400843@hospital.cqmu.edu.cn

Mrs. Juan Tan, Outpatient Department, The Children's Hospital of Chongqing Medical University, Lijia Campus, 20 Jinyu Avenue, Chongqing 401122, P.R. China

E-mail: 438570070@qq.com

${ }^{*}$ Contributed equally

Key words: long non-coding RNA, X-inactive specific transcript, meta-analysis, cancer, clinical outcome
However, sex was not associated with lncRNA-XIST expression level. In the OS patient cohort, it was demonstrated that lncRNA-XIST was highly expressed in OS tissues, which negatively correlated with patient prognosis. The present study indicated that IncRNA-XIST may serve as a potential biomarker for advanced clinical parameters in human cancer.

\section{Introduction}

Cancer is one of the leading causes of mortality worldwide (1). Although progress in cancer treatment has been achieved in previous decades, the prognosis for the majority of cancer types remains poor. Early diagnosis and treatment are important in improving the prognosis of patients with cancer. However, the sensitivity and specificity of the cancer markers used currently are not satisfactory (2). Therefore, novel molecular markers for predicting advanced cancer status and prognosis are required.

Although $70-80 \%$ of the human genome is transcribed into RNA, protein-coding sequences account for a small fraction of the total transcripts, indicating that the number of non-coding RNAs is increased compared with that of protein-coding genes (3-6). Specifically, long noncoding RNAs (lncRNAs) constitute a class of non-coding RNAs measuring $>200$ nucleotides in length, with no protein-coding capacity. These lncRNAs serve key roles in chromatin regulation, gene expression, growth, differentiation and development (7). Although the existence of lncRNAs has been known for some time, the term 'IncRNA' was not adopted until more recently. There are a large number of IncRNAs included in numerous databases, including LNCipedia (http://www.Incipedia.org) and the NONCODE database (http://www.noncode.org). As this number continues to increase, there are more opportunities to investigate the functions of these noncoding elements, particularly in association with cancer treatment and prognosis (8).

Accumulating evidence has suggested an oncogenic or tumor suppressive role for IncRNAs during tumorigenesis, in which numerous IncRNAs have been revealed to be dysregulated $(9,10)$. IncRNA-X-inactive specific transcript (XIST) 
was the first functional lncRNA identified to be responsible for X-chromosome inactivation (11). A growing body of data has revealed that IncRNA-XIST behaves in an oncogenic manner in colorectal (12), bladder (13) and gastric cancer (14), in addition to nasopharyngeal cancer (15) and osteosarcoma (OS) (16).

By contrast, XIST has been demonstrated to serve a tumor suppressor role in other studies (17-19). However, the majority of the data described so far is limited by discrete outcomes and small sample sizes. In recent years, several meta-analyses were conducted to evaluate the prognostic value of IncRNA-XIST in patients with cancer, and it was identified that the expression level of XIST was associated with overall survival, lymph node metastasis, distant metastasis and tumor stage (20-22). However, following these meta-analyses, a number of studies concerning XIST and cancer were published, with some describing contradicting conclusions $(23,24)$. Therefore, we propose that an updated meta-analysis is required. In order to assess the value of IncRNA-XIST in predicting the progression of clinicopathological features in patients with cancer, a meta-analysis was conducted and a case series of 45 patients with OS was described.

\section{Patients and methods}

Search strategy. To identify the incidence of lncRNA-XIST expression in cancer, PubMed (https://www.ncbi.nlm.nih. gov/pubmed), Web of Science (www.webofknowledge. com/), Embase (https://www.embase.com/) and the Cochrane Library (https://www.cochranelibrary.com/) databases were searched for articles published prior to January 2019, using the search terms 'long non-coding RNA' OR 'IncRNA,' AND 'cancer' OR 'sarcoma' OR 'carcinoma' OR 'neoplasm' OR 'malignancy', AND 'XIST'. Additionally, reference lists of associated reviews were searched to identify any potentially relevant studies. The inclusion criteria were as follows: i) The publication explored the relevance of lncRNA-XIST expression in human tumor tissues; ii) high and low lncRNA-XIST expression groups were defined, or the relevant data to categorize patients into these groups was present; and iii) the publication language was confined to English. The following articles were excluded from the study i) Reviews, editorials, meetings, abstracts and commentaries; ii) publications with no target data or relevant outcomes; and iii) duplicate studies.

The following basic information was extracted from each study using a standardized data collection method: i) First authors; ii) publication year; iii) study population; iv) sample size; v) tumor type; and vi) lncRNA-XIST detection method. If any essential information was not available from the original article, best efforts were made to contact the corresponding author to obtain the missing data. As summarized in Table I, all of the included publications were evaluated based on the critical checklist of the Dutch Cochrane Centre, as proposed by Meta-analysis of Observational Studies in Epidemiology (25).

Evaluation of clinical samples. A total of 45 (25 females and 20 males) paired OS tissues and adjacent healthy tissues from patients aged from 9-14 years (from January 2009 to September 2017), with no preoperative history of radiotherapy and/or chemotherapy were obtained from the Children's
Hospital of Chongqing Medical University (Chongqing, China). The tumor and adjacent healthy tissues were obtained during biopsy/resection prior to chemotherapy. Staging was performed based on the Musculoskeletal Tumor Society staging system (26). Following resection, the tissues were immediately frozen in liquid nitrogen. The present study was performed with the approval of the Institutional Review Board of Children's Hospital of Chongqing Medical University. Written informed consent was obtained from the parents of all patients.

Total RNA was isolated with TRIzol ${ }^{\circledR}$ reagent (Thermo Fisher Scientific, Inc.) according to the manufacturer's protocol and subjected to reverse transcription (RT) reactions using hexamers, dNTPs and M-MuLV Reverse Transcriptase (supplied with 10X Reaction Buffer) (New England BioLabs, Inc.). The thermocycling conditions of the RT polymerase chain reaction (RT-PCR) were as follows: $37^{\circ} \mathrm{C}$ for $60 \mathrm{~min}$, then at $95^{\circ} \mathrm{C}$ for $1 \mathrm{~min}$, followed by holding at $4^{\circ} \mathrm{C}$. The resultant cDNA products were diluted 10 - to 100 -fold and used as templates. RT-quantitative PCR (RT-qPCR) analysis was performed using the optimized touchdown $\mathrm{qPCR}$ protocol described previously by Zhang et al (27). Briefly, the SYBR Green qPCR reactions (Bio-Rad Laboratories, Inc.) were performed in triplicate, according to manufacturer's protocol, under the following thermocycler conditions: $95^{\circ} \mathrm{C}$ for $3 \mathrm{~min}$, followed by $95^{\circ} \mathrm{C}$ for $20 \mathrm{sec}$ and $66^{\circ} \mathrm{C}$ for $10 \mathrm{sec}$ for 4 cycles (decreasing by $3^{\circ} \mathrm{C}$ per cycle); then $95^{\circ} \mathrm{C}$ for $20 \mathrm{sec}, 55^{\circ} \mathrm{C}$ for $10 \mathrm{sec}$ and $70^{\circ} \mathrm{C}$ for $1 \mathrm{sec}$, for 40 cycles. The $2^{-\Delta \Delta \mathrm{Cq}}$ method was used to determine the relative quantitation of lncRNA-XIST expression levels (28). The primer sequences used in were as followed: GAPDH forward, 5'-GTCAAGGCTGAGAACGGG AA-3'; GAPDH reverse, 5'-AAATGAGCCCCAGCCTTC TC-3'; IncRNA-XIST forward, 5'-GGTGGACATGTGCGG TCA-3'; and IncRNA-XIST reverse, 5'-CCTGCGGCAAAA CCCAAC -3 '.

Statistical analysis. All statistical analyses were performed using Stata 12.0 (StataCorp LP) and Revman5.2 software (Cochrane). The combined odds ratio (OR) and 95\% confidence interval (CI) were used to determine the association between lncRNA-XIST expression level and clinical risk. The combined effect size was statistically significant when it did not overlap with 1 . Heterogeneity across the studies was quantified using the $\mathrm{I}^{2}$ statistic. A fix-effects model with the inverse variance method was conducted when the calculated $\mathrm{I}^{2}<50 \%(29,30)$. If $\mathrm{I}^{2}>50 \%$, subgroup analysis was performed. Potential publication bias was assessed using Egger's bias indicator test with the linear regression method, respectively, and sensitivity analysis was also conducted. For analysis of the clinical samples, the patients were divided into two groups according to the expression level of lncRNA-XIST (the median expression level 2.4 was used as the cut-off). The $\chi^{2}$ test was used to identify the differences between categorical variables, and the two-tailed Student's t-test was used for comparisons between groups. Overall survival was estimated with Kaplan-Meier method. $\mathrm{P}<0.05$ was considered to indicate a statistically significant difference.

Quality assessment. The quality of each study was assessed using the Newcastle-Ottawa Scale (31), consisting of three 
Table I. Characteristics of the studies included.

\begin{tabular}{|c|c|c|c|c|c|c|c|c|}
\hline First author & Year & Country & $\begin{array}{l}\text { Sample } \\
\text { size }\end{array}$ & Ethnicity & $\begin{array}{l}\text { Cancer } \\
\text { type }\end{array}$ & $\begin{array}{l}\text { Detection } \\
\text { method }\end{array}$ & $\begin{array}{l}\text { Quality } \\
\text { assessment } \\
\text { score }\end{array}$ & (Refs.) \\
\hline Tantai & 2015 & China & 32 & Asian & NSCLC & qPCR & 6 & (32) \\
\hline Kobayashi & 2016 & Japan & 49 & Asian & $\begin{array}{l}\text { Cervical squamous } \\
\text { cell carcinoma }\end{array}$ & qPCR & 5 & (19) \\
\hline Fang & 2016 & China & 53 & Asian & NSCLC & qPCR & 7 & (33) \\
\hline Chen & 2016 & China & 106 & Asian & Gastric cancer & qPCR & 5 & (34) \\
\hline $\mathrm{Li}$ & 2017 & China & 145 & Asian & Osteosarcoma & qPCR & 6 & (35) \\
\hline Chen & 2017 & China & 115 & Asian & Colorectal cancer & qPCR & 8 & (36) \\
\hline Wei & 2017 & China & 64 & Asian & Pancreatic cancer & qPCR & 7 & (37) \\
\hline $\mathrm{Du}$ & 2017 & China & 69 & Asian & Glioma & qPCR & 7 & $(38)$ \\
\hline Wang & 2017 & China & 30 & Asian & Glioma & qPCR & 7 & (39) \\
\hline Song & 2017 & China & 50 & Asian & Colorectal cancer & qPCR & 6 & (12) \\
\hline $\mathrm{Ma}$ & 2017 & China & 98 & Asian & Gastric cancer & qPCR & 8 & (14) \\
\hline Мо & 2017 & China & 88 & Asian & Hepatocellular carcinoma & qPCR & 7 & $(40)$ \\
\hline Sun & 2017 & China & 47 & Asian & Cervical cancer & qPCR & 6 & (41) \\
\hline Xiong & 2017 & China & 67 & Asian & Bladder cancer & qPCR & 6 & (42) \\
\hline Sun & 2017 & China & 50 & Asian & NSCLC & qPCR & 6 & (43) \\
\hline $\mathrm{Du}$ & 2017 & China & 62 & Asian & Prostate cancer & qPCR & 7 & (18) \\
\hline $\mathrm{Hu}$ & 2017 & China & 52 & Asian & Bladder cancer & qPCR & 7 & (13) \\
\hline $\mathrm{Wu}$ & 2017 & China & 127 & Asian & $\begin{array}{l}\text { Esophageal squamous } \\
\text { cell carcinoma }\end{array}$ & qPCR & 6 & $(44)$ \\
\hline Kong & 2018 & China & 52 & Asian & Hepatocellular carcinoma & qPCR & 7 & (45) \\
\hline Yi & 2018 & China & 140 & Asian & $\begin{array}{l}\text { Esophageal squamous } \\
\text { cell carcinoma }\end{array}$ & qPCR & 7 & $(46)$ \\
\hline Liang & 2017 & China & 73 & Asian & Pancreatic carcinoma & qPCR & 7 & (47) \\
\hline Sun & 2018 & China & 120 & Asian & Colon cancer & qPCR & 7 & $(48)$ \\
\hline Liu & 2018 & China & 77 & Asian & Thyroid cancer & qPCR & 7 & (49) \\
\hline $\mathrm{Hu}$ & 2018 & China & 30 & Asian & Retinoblastoma & qPCR & 6 & $(50)$ \\
\hline Zhu & 2018 & China & 52 & Asian & Cervical cancer & qPCR & 7 & (51) \\
\hline
\end{tabular}

NSCLC, non-small cell lung cancer; NA, not available; qPCR, quantitative polymerase chain reaction.

parts: Selection; outcome; and comparability, with a score range of $0-9$. A score $\geq 6$ was considered to indicate high quality.

\section{Results}

Literature analysis. A total of 770 citations were retrieved from an initial online search for literature associated with IncRNA-XIST expression in cancer. A total of 644 citations were excluded following initial screening of titles and abstracts; of the remaining 125 candidate studies (which were reviewed in their entirety), 100 were also excluded. A total of 25 studies were included in the final analysis (Fig. 1).

Study characteristics. The characteristics of the final 25 articles are presented in Table I (12-14,18,19,32-51). These studies were published between 2015 and 2018, with sample sizes of between 31-146 patients. A total of 1,869 patients were divided into two groups (high and low expression of
lncRNA-XIST) according to RT-qPCR results. The majority of the studies were conducted in China and the patients presented with the following 12 cancer types: Hepatocellular carcinoma; gastric cancer; pancreatic cancer; osteosarcoma; cervical cancer; bladder cancer; esophageal squamous cell carcinoma; glioma; colorectal cancer; non-small-cell lung cancer (NSCLC); thyroid cancer; retinoblastoma; and cervical cancer.

IncRNA-XIST expression and patient outcome. As outlined in Table II, the results demonstrated that high expression levels of lncRNA-XIST were associated with lymphatic metastasis (OR =2.32; 95\% CI 1.81-3.00; $\mathrm{P}=0.028$; Fig. 2), larger tumor size $(\mathrm{OR}=2.60 ; 95 \%$ CI 1.91-3.56; $\mathrm{P}=0.001$; Fig. 3), advanced cancer stage $(\mathrm{OR}=2.97 ; 95 \%$ CI $2.15-4.09 ; \mathrm{P}=0.001$; Fig. 4) and positive distant metastasis $(\mathrm{OR}=2.07$; 95\% CI 1.24-3.47; $\mathrm{P}=0.001$; Fig. 5). However, sex $(\mathrm{OR}=0.96$; 95\% CI 0.79-1.18; $\mathrm{P}=0.916)$ was not associated with lncRNA-XIST expression level (Fig. 6). 
Table II. Primary outcome of X-inactive specific transcript expression on disease characteristics.

\begin{tabular}{lccccrrr}
\hline & & & & & & \multicolumn{2}{c}{ Egger's test } \\
\cline { 5 - 8 } Disease characteristics & OR $(95 \% \mathrm{CI})$ & Z-value & P-value & $\mathrm{P}_{\text {het }}$ & $\mathrm{I}^{2}(\%)$ & $\mathrm{t}$ & P-value \\
\hline Lymph node metastasis & & & & & & & \\
(yes vs. no) & $2.93(2.09,4.10)$ & 6.27 & $<0.001$ & 0.37 & 7 & -0.47 & 0.65 \\
Tumour size (bigger vs. smaller) & $3.07(2.40,3.92)$ & 8.98 & $<0.001$ & 0.16 & 26 & -0.57 & 0.58 \\
Stage (III/IV vs. I/II) & $2.86(1.85,4.42)$ & 4.72 & $<0.001$ & 0.00 & 64 & 1.89 & 0.08 \\
Distant metastasis (yes vs. no) & $1.76(0.76,4.08)$ & 3.59 & $<0.001$ & 0.64 & 79 & -1.77 & 0.15 \\
Sex (female vs. male) & $1.02(0.79,1.30)$ & 0.13 & 0.89 & 0.57 & 0 & 0.71 & 0.49 \\
\hline
\end{tabular}

OR, odds ratio; $\mathrm{CI}$, confidence interval; $\mathrm{P}_{\text {het }}$, heterogeneity.

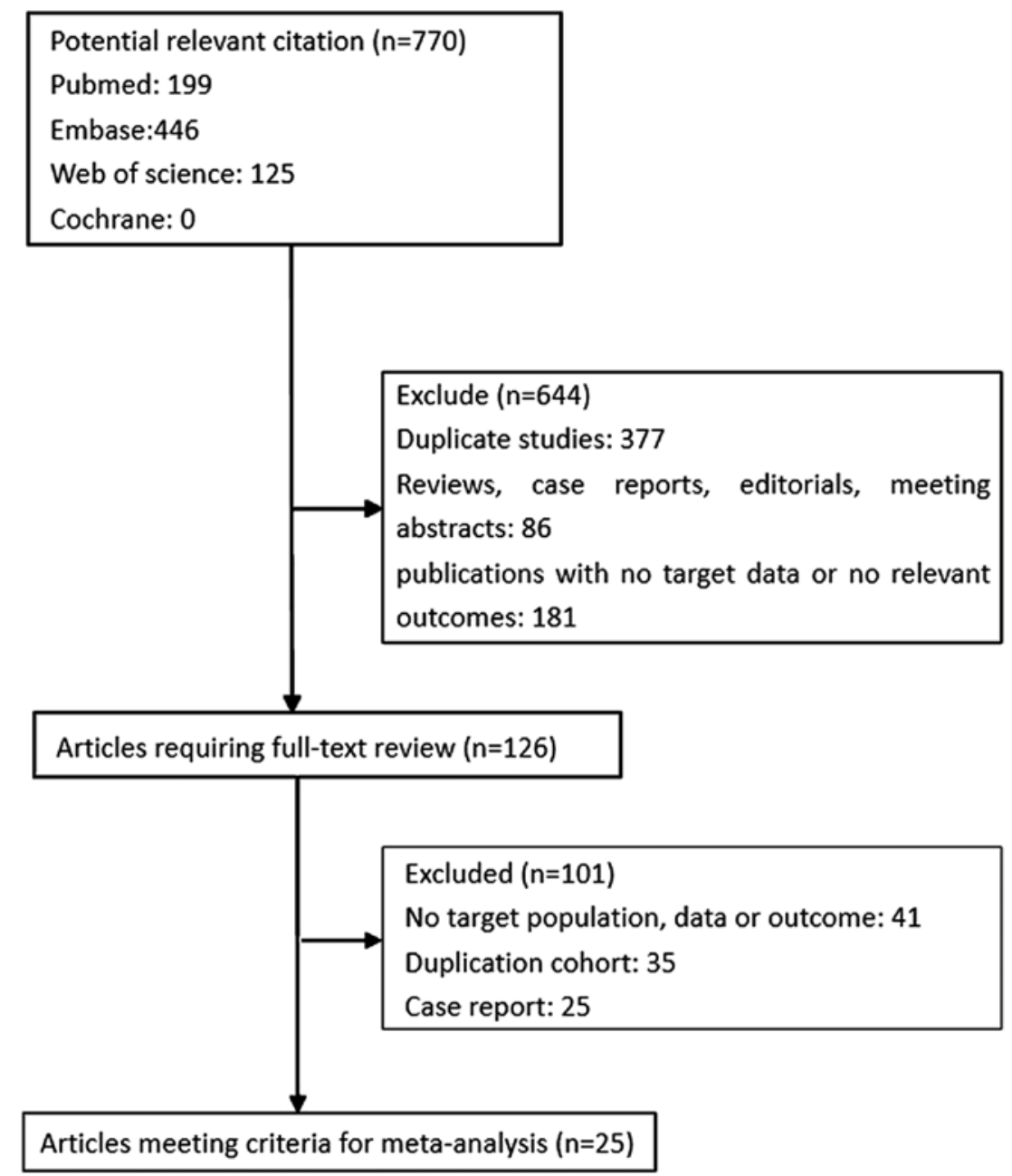

Figure 1. Flow diagram of meta-analysis process. A total of 644 publications were excluded following screening of titles and abstracts. Then, 25 eligible articles were identified to meet the criteria for the meta-analysis from the 126 relevant articles included in the full-text selection.

Publication bias and sensitivity analysis. The Egger linear regression test indicated a potential publication bias in the stage category (Table II). Next, sensitivity analysis was performed to evaluate the stability of the present study. Each parameter was excluded from the sensitivity analysis, and the results of our meta-analysis were consistent, indicating that the combined results were stable (Figs. S1-S5). Due to the marked heterogeneity observed, subgroup analysis was performed for distant metastasis, stage and tumor size. From the Figs. S6-S9, subgroup analysis results of cancer types, publication year, sample size and quality assessment for distant metastasis demonstrated that the levels of heterogeneity had not decreased, indicating that cancer types, publication year, sample size and quality assessment were not the source of heterogeneity. The 


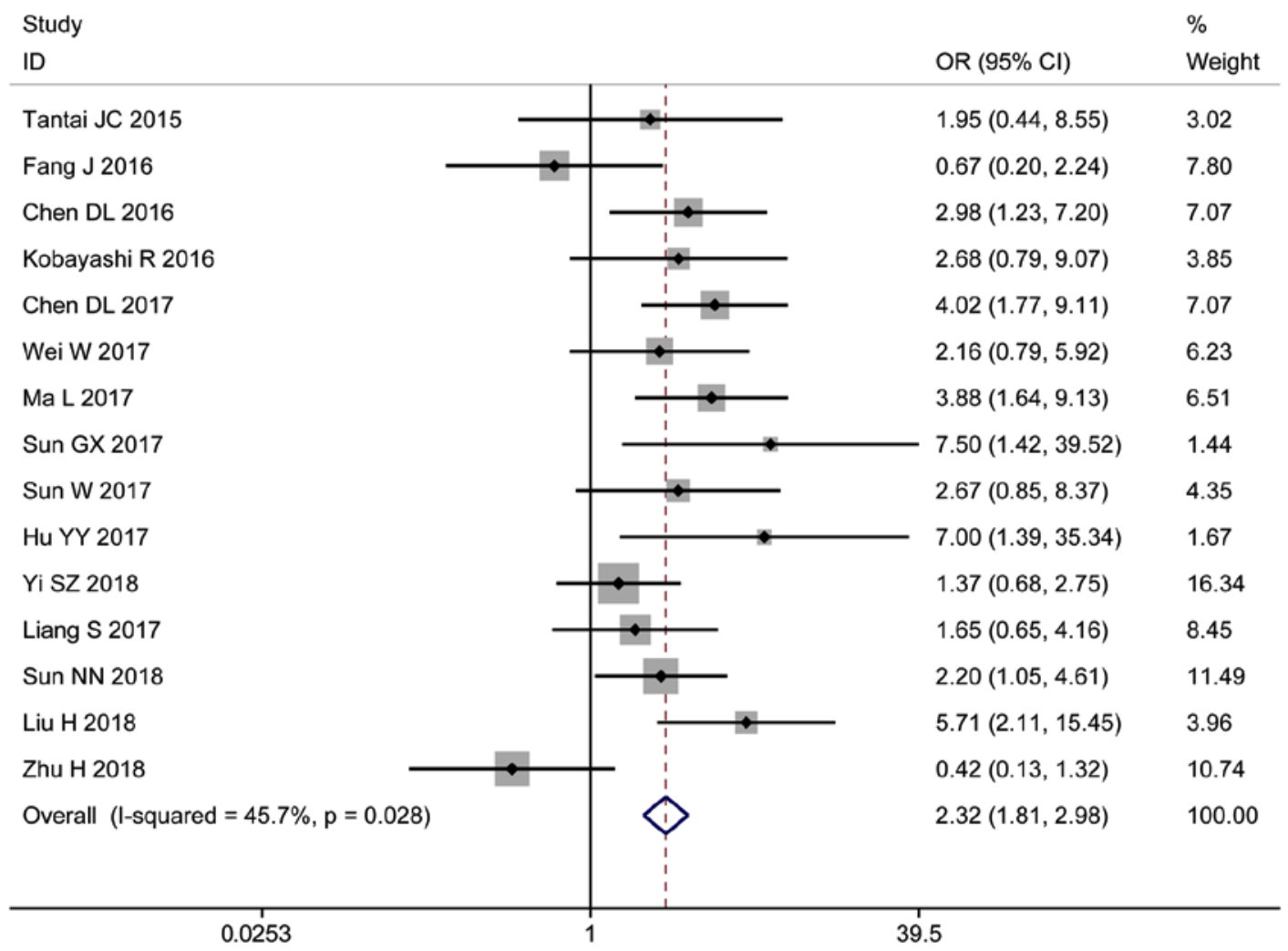

Figure 2. Forest plot studies investigating the association between long non-coding RNA X-inactive specific transcript and lymph node metastasis in a fixed effect model. OR, odds ratio; CI, confidence interval.

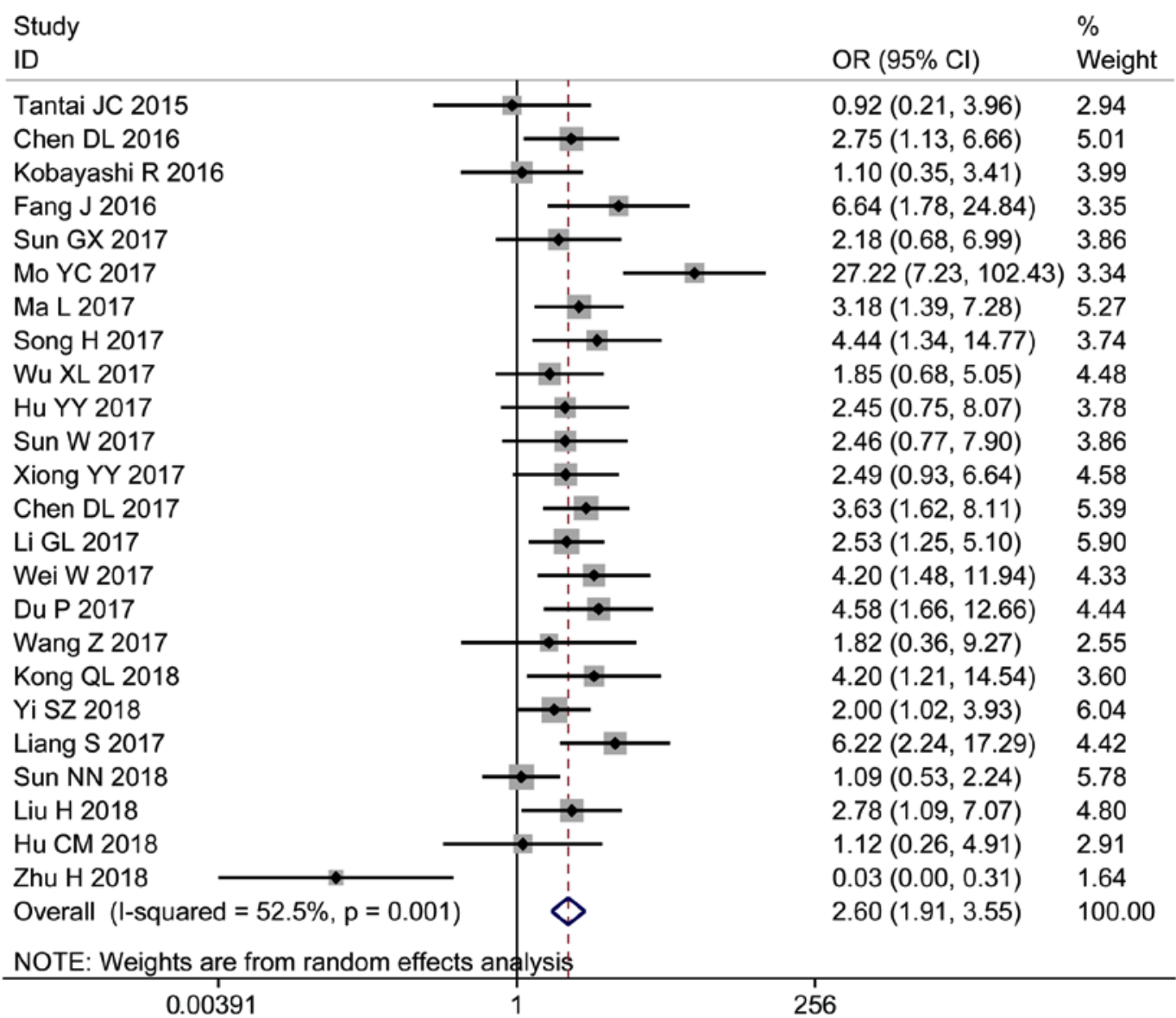

Figure 3. Forest plot of studies examining the association between long non-coding RNA X-inactive specific transcript and tumor size in a random effect model. OR, odds ratio; CI, confidence interval. 


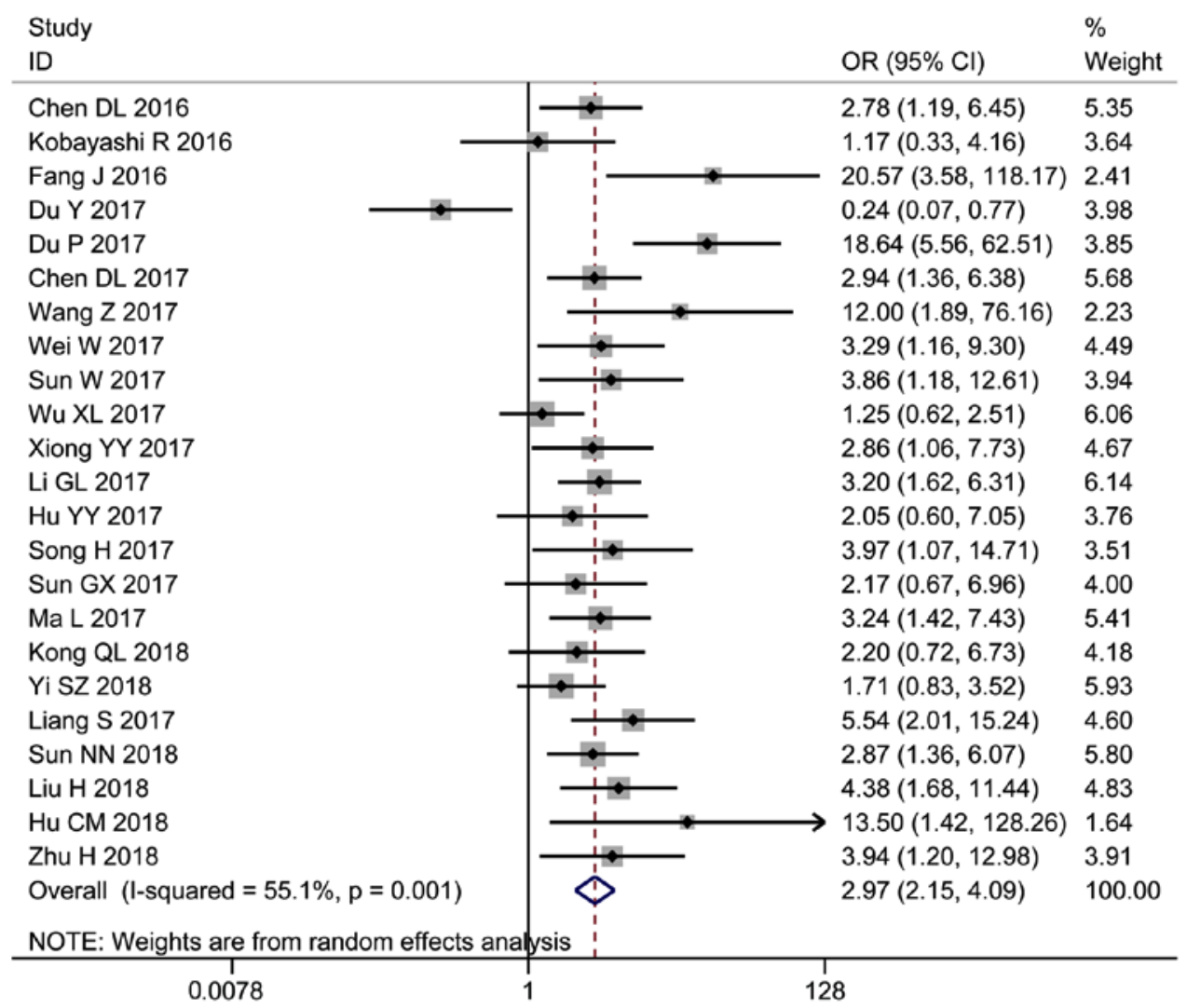

Figure 4. Forest plot of studies investigating the association between long non-coding RNA X-inactive specific transcript and disease stage in a random effects model. OR, odds ratio; $\mathrm{CI}$, confidence interval.

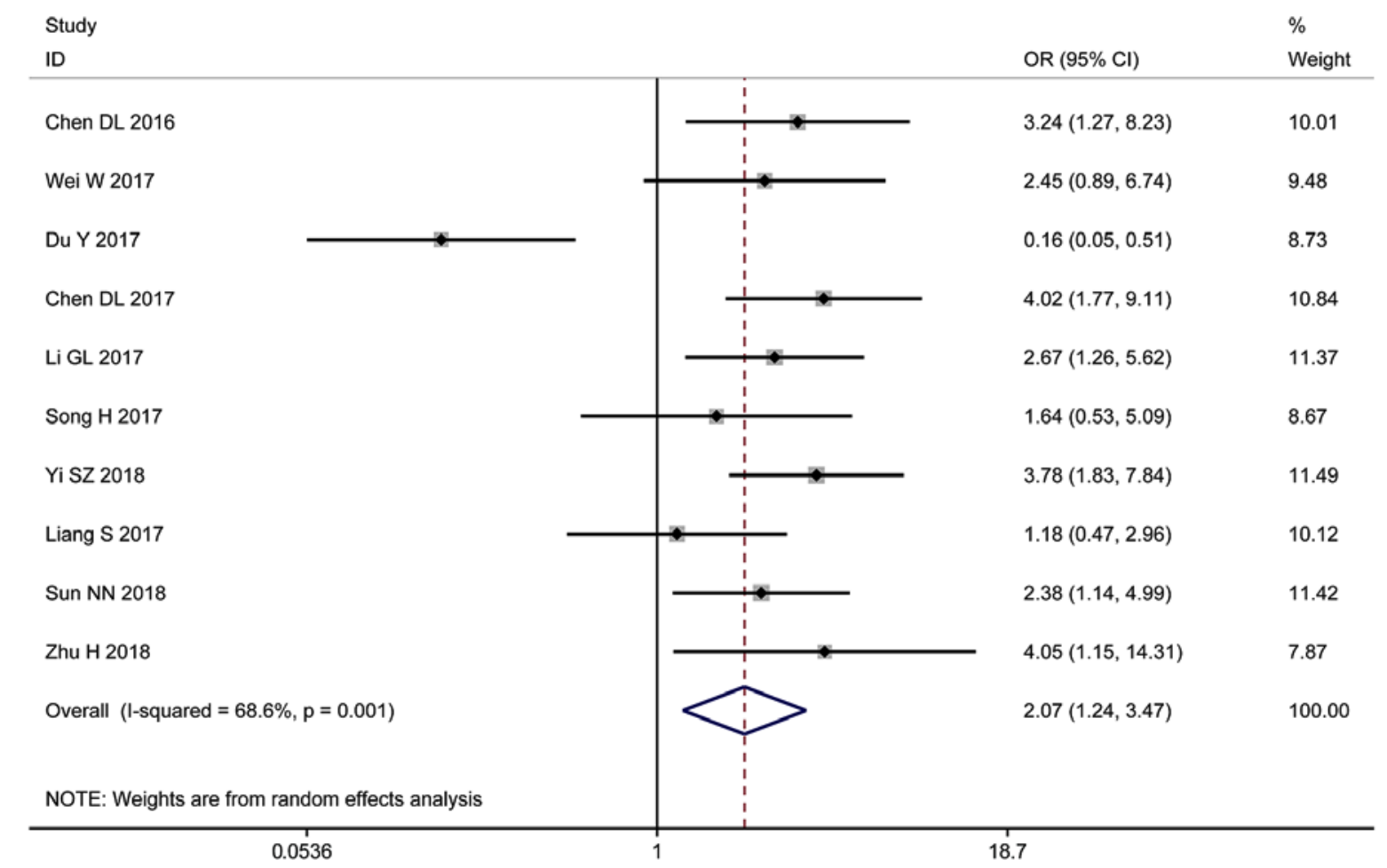

Figure 5. Forest plot of studies examining the association between long non-coding RNA X-inactive specific transcript and distant metastasis in a random effect model. OR, odds ratio; CI, confidence interval.

same results were identified in tumor size or stage subgroup analyses (Figs. S10-S17).
Quality assessment. The scores of the included studies ranged between 5-8, as determined using the Newcastle-Ottawa Scale. 


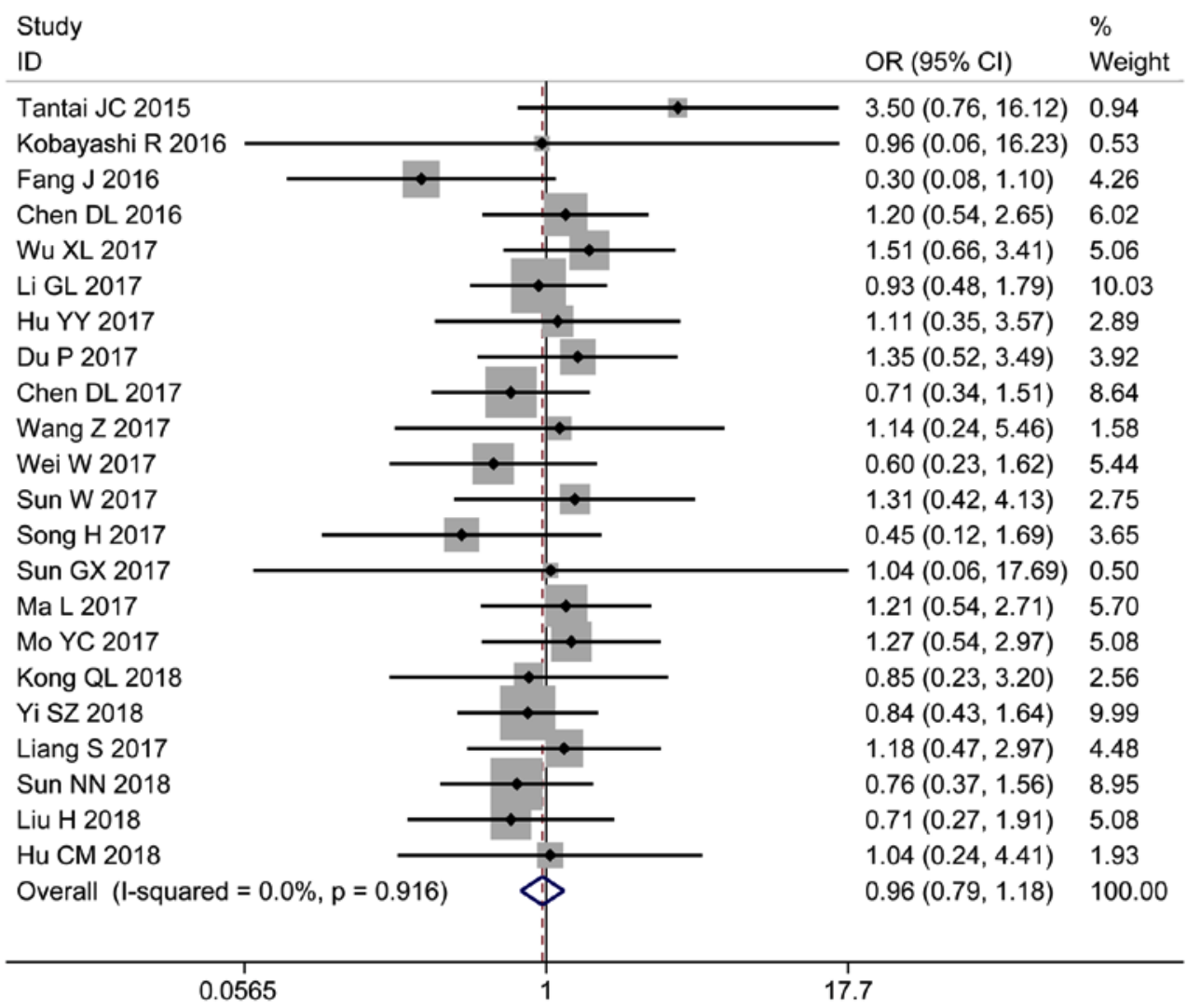

Figure 6. Forest plot of studies investigating the association between long non-coding RNA X-inactive specific transcript and sex in a fixed effect model. OR, odds ratio; $\mathrm{CI}$, confidence interval.
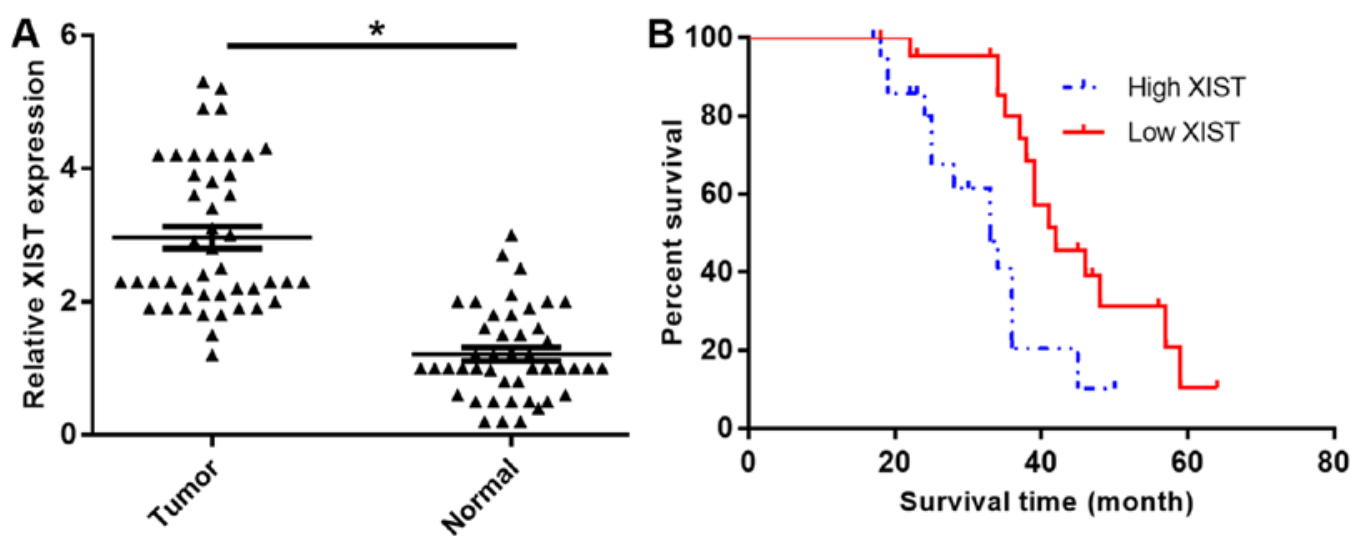

Figure 7. Validation of XIST role in the OS sample from the present study. (A) Reverse transcription quantitative polymerase chain reaction analysis demonstrated that the levels of XIST were significantly up-regulated in OS tumor samples compared with normal adjacent tissues. (B) Kaplan-Meier analysis indicated that high expression of XIST was inversely associated with overall survival in patients with OS. "P<0.05. OS, overall survival; XIST, X-inactive specific transcript.

The majority of the studies scored $>7 ; 15$ studies scored 7 and 2 studies scored 8 (Table I).

Clinical sample confirmation. To determine the role of IncRNA-XIST in patients with OS, expression levels were determined in the cancerous and adjacent healthy tissues of patients using RT-qPCR (Fig. 7A). The results revealed that lncRNA-XIST expression level was significantly upregulated in OS (Fig. 7A), and that high expression levels were inversely associated with patient overall survival (Fig. 7B). The potential association between IncRNA-XIST expression level and patient clinicopathological features was also evaluated (Table III). The results revealed that high IncRNA-XIST expression level correlated with advanced clinical stage and tumor size.

\section{Discussion}

Cancer is one of the leading causes of mortality worldwide (52). The identification of novel biomarkers is necessary for early diagnosis, and to improve the prognosis of patients with cancer. An increasing number of studies have suggested that lncRNAs are aberrantly expressed in various types of human cancer; 
Table III. Correlations between clinicopathological features and the expression of XIST in osteosarcoma tissues.

\begin{tabular}{|c|c|c|c|c|}
\hline \multirow[b]{2}{*}{ Clinicopathological features } & \multirow[b]{2}{*}{ Number of patients } & \multicolumn{2}{|c|}{ XIST expression } & \multirow[b]{2}{*}{ P-value } \\
\hline & & High & Low & \\
\hline \multicolumn{5}{|l|}{ Sex } \\
\hline Male & 22 & 12 & 10 & 0.87 \\
\hline Female & 23 & 12 & 11 & \\
\hline Ages, years [Mean (SD)] & & $13.2(2.4)$ & $12.7(2.9)$ & 0.29 \\
\hline \multicolumn{5}{|l|}{ Histological type } \\
\hline Osteoblastic & 31 & 17 & 14 & 0.76 \\
\hline Chondroblastic & 14 & 7 & 7 & \\
\hline \multicolumn{5}{|l|}{ TNM stage } \\
\hline I, II & 23 & 8 & 15 & 0.01 \\
\hline III & 22 & 16 & 6 & \\
\hline \multicolumn{5}{|l|}{ Tumor size, $\mathrm{cm}$} \\
\hline$<5$ & 18 & 6 & 12 & 0.02 \\
\hline$>5$ & 27 & 18 & 9 & \\
\hline
\end{tabular}

XIST, X-inactive specific transcript; TNM, tumor node metastasis.

furthermore, an association between IncRNA expression, pathophysiological features and patient survival has also been indicated, making lncRNAs promising biomarkers for cancer prognosis (53). It has also been demonstrated that lncRNAs may exert their functions via transcription and epigenetic regulation, as they also serve as scaffolds in the formation of ribonucleoprotein complexes (54). Various lncRNAs, including IncRNA-maternally expressed 3 (55), lncRNA-colon cancer associated transcript 2 (56) and lncRNA-HOX transcript antisense RNA (57) have been identified as novel indicators of poor prognosis in a number of types of human cancer.

Accumulating evidence implies a regulatory role for IncRNA-XIST, the earliest identified lncRNA, in various malignant tumors. Wei et al (37) demonstrated that IncRNA-XIST was involved in the proliferation, invasion, and epithelial-mesenchymal transition of cancer cells. Additionally, Wang et al (39) suggested that IncRNA-XIST promoted glioma cell proliferation by targeting microRNA-137. A study by Chen et al (34) indicated that high IncRNA-XIST expression levels were positively correlated with aggressive tumor phenotypes and prognosis in gastric cancer, and enhanced the functions of enhancer of zeste homolog 2. In nasopharyngeal carcinoma, abnormal expression of 1ncRNA-XIST was revealed to promote cell proliferation, partially by suppressing miRNA-34a-5p, and subsequently activating E2F transcription factor 3 (15). Small interfering RNA inhibition of lncRNA-XIST suppressed the proliferation, migration and invasion of NSCLC cells in vitro, and suppressed tumor growth in vivo (33). Based on these data, IncRNA-XIST may be a potential prognostic marker for patients with cancer (35-38).

Conversely, IncRNA-XIST may serve as a tumor suppressor in specific types of cancer. Kobayashi et al (19) revealed that increased expression levels of lncRNA-XIST were positively associated with favorable prognosis in cervical squamous cell carcinoma. In breast cancer, a decreased expression of
IncRNA-XIST upregulated the phosphorylation of protein kinase B and inhibited tumor growth (17). Based on these contradictory results, the true value of IncRNA-XIST as a tumor marker remains to be determined. It is necessary to comprehensively evaluate the clinical significance of lncRNA-XIST. Therefore, meta-analyses were conducted to evaluate the prognostic value of IncRNA-XIST in patients with cancer. The study conducted by $\mathrm{Hu}$ et al (20) examined 9 studies with 853 patients with cancer, and identified that the expression level of IncRNA-XIST was markedly associated with overall survival, disease free survival, tumor type, lymph node metastasis, distant metastasis and tumor stage. Mao et al (21) identified 15 eligible studies containing 1,209 patients for inclusion in their meta-analysis; they observed that increased lncRNA-XIST expression levels in cancer tissues were associated with a poorer overall survival. In the study conducted by Liu et al (22), 15 studies with a total of 920 patients were included in the meta-analysis, and the results suggested that high lncRNA-XIST expression levels were associated with distant metastasis, tumor stage and poor prognosis. However, a number of contrasting studies were published concerning the role of IncRNA-XIST in cancer: In a recent study by Du et al (18), lncRNA-XIST served as a tumor suppressor in prostate cancer, its expression correlating with prognosis and tumor stage. In addition, Sun et al (23) identified that lncRNA-XIST regulated the microRNA-106b-5p/cyclin-dependent kinase inhibitor 1 axis to suppress tumor progression in renal cell carcinoma. Consequently, we proposed that an updated meta-analysis was required. In the present study, a comprehensive and detailed meta-analysis was conducted to investigate the association between IncRNA-XIST expression and the clinicopathological characteristics of patients with cancer. The analysis of 25 studies, including 1,869 cancer patients, indicated that high lncRNA-XIST expression level was significantly associated with lymphatic metastasis, larger tumor size, advanced stage 
and positive distant metastasis, suggesting that increased lncRNA-XIST levels may be associated with advanced disease presentation. It should also be noted that the prognostic value of IncRNA-XIST may vary between different types of cancer. For example, the expression level of lncRNA-XIST was significantly associated with tumor size in all types of cancer in the included studies, with the exception of NSCLC and cervical squamous cell carcinoma.

As a number of the clinicopathological characteristics of cancer may be associated with the sex of the patients, and IncRNA-XIST is responsible for X-chromosome inactivation, it was speculated that there may be a sex-specific association between the expression of IncRNA-XIST and the features of different types of cancer. Although the results of the present study revealed no significant association between IncRNA-XIST expression and sex, this should be considered in future studies.

Following a review of the current literature, potential associations between clinicopathological features and the expression of IncRNA-XIST in OS tissues were determined. It was confirmed that IncRNA-XIST expression was upregulated in OS tissues, and that high expression levels were inversely correlated with the overall survival of patients with OS. Advanced staging and increased tumor size were closely associated with increased lncRNA-XIST expression levels. The results of the present study were consistent with the conclusions of meta-analysis, additionally highlighting the importance of IncRNA-XIST in human malignancies.

In the present study, a number of limitations should be considered. Only papers written in English were included. Additionally, the majority of the studies originated from China, therefore the results are largely representative of the Chinese population. Furthermore, the cut-off values for high and low expression levels of IncRNA-XIST differed between the studies. Also, due to the small number of studies comparing the expression of IncRNA-XIST with distant metastasis, the significance of certain data may have been limited by population size. Positive results were described in the majority of studies, whilst those with negative results were less likely to be published, which may be suggestive of a publication bias. Also, the association between IncRNA-XIST expression and the survival rates of patients was not determined. However, recent studies revealed that high expression levels were correlated with decreased overall and disease-free survival $(20,22,58)$. The subgroup analysis did not identify a source of heterogeneity, although significant heterogeneity in distant metastasis, tumor size and stage was observed. More high-quality original studies are required to confirm the conclusions of the present study.

Given the aforementioned limitations, the present study supports the hypothesis that the upregulation of IncRNA-XIST expression may be considered a credible predictive factor for advanced clinicopathological features in human cancer. In the future, large-scale and multicenter studies are required to confirm these results, and to validate the clinical significance of lncRNA-XIST expression in human cancer.

\section{Acknowledgements}

The authors would like to thank Dr T.-C. He and Ms Mia Spezia of The University of Chicago Medical Center for their critical analysis of the manuscript.

\section{Funding}

The present study was supported by a research grant from Chongqing Science and Technology Commission (grant no. cstc2015jcyjA10046) and the Chengdu Women's and Children's Central Hospital of Chongqing Medical University Research Projects (Project No.: 1712).

\section{Availability of data and materials}

All data generated or analyzed during this study are included in this published article.

\section{Authors' contributions}

CY and JT conceived and designed the study, acquired data, and drafted the manuscript. CD and CY performed the acquisition of data and analyzed the data. XH and KW acquired data.

\section{Ethics approval and consent to participate}

The present study was performed with the approval of the Institutional Review Board of Children's Hospital of Chongqing Medical University. Written informed consent was obtained from the parents of all patients.

\section{Patient consent for publication}

Written informed consent was obtained from the parents of all patients.

\section{Competing interests}

All authors declare that they have no competing interests.

\section{References}

1. Torre LA, Siegel RL, Ward EM and Jemal A: Global cancer incidence and mortality rates and trends-an update. Cancer Epidemiol Biomarkers Prev 25: 16-27, 2016.

2. Cui Z, Chen Y, Xiao Z, Hu M, Lin Y, Chen Y and Zheng Y: Long noncoding RNAs as auxiliary biomarkers for gastric cancer screening: A pooled analysis of individual studies. Oncotarget 7: 25791-800, 2016.

3. ENCODE ProjectConsortium,Birney E,Stamatoyannopoulos JA, Dutta A, Guigó R, Gingeras TR, Margulies EH, Weng Z, Snyder M, Dermitzakis ET, et al: Identification and analysis of functional elements in $1 \%$ of the human genome by the ENCODE pilot project. Nature 447: 799-816, 2007.

4. Kapranov P, Willingham AT and Gingeras TR: Genome-wide transcription and the implications for genomic organization. Nat Rev Genet 8: 413-423, 2007.

5. ENCODE Project Consortium: An integrated encyclopedia of DNA elements in the human genome. Nature 489: 57-74, 2012.

6. Sana J, Faltejskova P, Svoboda M and Slaby O: Novel classes of non-coding RNAs and cancer. J Transl Med 10: 103, 2012.

7. Tsai MC, Spitale RC and Chang HY: Long intergenic noncoding RNAs: New links in cancer progression. Cancer Res 71: 3-7, 2011.

8. Blythe AJ, Fox AH and Bond CS: The ins and outs of lncRNA structure: How, why and what comes next. Biochim Biophys Acta 1859: 46-58, 2016.

9. Tseng YY, Moriarity BS, Gong W, Akiyama R, Tiwari A, Kawakami H, Ronning P, Reuland B, Guenther $\mathrm{K}$, Beadnell TC, et al: PVT1 dependence in cancer with MYC copy-number increase. Nature 512: 82-86, 2014. 
10. Huang J, Zhou N, Watabe K, Lu Z, Wu F, Xu M and Mo YY: Long non-coding RNA UCA1 promotes breast tumor growth by suppression of p27 (Kip1). Cell Death Dis 5: e1008, 2014.

11. Brown CJ, Ballabio A, Rupert JL, Lafreniere RG, Grompe M, Tonlorenzi R and Willard HF: A gene from the region of the human $\mathrm{X}$ inactivation centre is expressed exclusively from the inactive X chromosome. Nature 349: 38-44, 1991.

12. Song H, He P, Shao T, Li Y, Li J and Zhang Y: Long non-coding RNA XIST functions as an oncogene in human colorectal cancer by targeting miR-132-3p. J BUON 22: 696-703, 2017.

13. Hu Y, Deng C, Zhang H, Zhang J, Peng B and Hu C: Long non-coding RNA XIST promotes cell growth and metastasis through regulating miR-139-5p mediated Wnt/ $\beta$-catenin signaling pathway in bladder cancer. Oncotarget 8: 94554-94568, 2017.

14. Ma L, Zhou Y, Luo X, Gao H, Deng X and Jiang Y: Long non-coding RNA XIST promotes cell growth and invasion through regulating miR-497/MACC1 axis in gastric cancer. Oncotarget 8: 4125-4135, 2017.

15. Song $\mathrm{P}$, Ye LF, Zhang $\mathrm{C}$, Peng $\mathrm{T}$ and Zhou $\mathrm{XH}$ : Long non-coding RNA XIST exerts oncogenic functions in human nasopharyngeal carcinoma by targeting miR-34a-5p. Gene 592: 8-14, 2016.

16. Yang $\mathrm{C}, \mathrm{Wu} \mathrm{K}$, Wang $\mathrm{S}$ and Wei G: Long non-coding RNA XIST promotes osteosarcoma progression by targeting YAP via miR-195-5p. J Cell Biochem 119: 5646-5656, 2018.

17. Huang YS, Chang CC, Lee SS, Jou YS and Shih HM: Xist reduction in breast cancer upregulates AKT phosphorylation via HDAC3-mediated repression of PHLPP1 expression. Oncotarget 7: 43256-43266, 2016

18. Du Y, Weng XD, Wang L, Liu XH, Zhu HC, Guo J, Ning JZ and Xiao CC: LncRNA XIST acts as a tumor suppressor in prostate cancer through sponging miR-23a to modulate RKIP expression. Oncotarget 8: 94358-94370, 2017.

19. Kobayashi R, Miyagawa R, Yamashita H, Morikawa T, Okuma K, Fukayama M, Ohtomo K and Nakagawa K: Increased expression of long non-coding RNA XIST predicts favorable prognosis of cervical squamous cell carcinoma subsequent to definitive chemoradiation therapy. Oncol Lett 12: 3066-3074, 2016.

20. Hu S, Chang J, Li Y, Wang W, Guo M, Zou EC, Wang Y and Yang Y: Long non-coding RNA XIST as a potential prognostic biomarker in human cancers: A meta-analysis. Oncotarget 9: 13911-13919, 2018

21. Mao H, Wang K, Feng Y, Zhang J, Pan L, Zhan Y, Sheng H and Luo G. Prognostic role of long non-coding RNA XIST expression in patients with solid tumors: A meta-analysis. Cancer Cell Int 18: 34, 2018.

22. Liu X, Ming X, Jing W, Luo P, Li N, Zhu M, Yu M, Liang C and $\mathrm{Tu} \mathrm{J}$ : Long non-coding RNA XIST predicts worse prognosis in digestive system tumors: A systemic review and meta-analysis. Biosci Rep 38: BSR20180169, 2018.

23. Sun K, Jia Z, Duan R, Yan Z, Jin Z, Yan L, Li Q and Yang J: Long non-coding RNA XIST regulates miR-106b-5p/P21 axis to suppress tumor progression in renal cell carcinoma. Biochem Biophys Res Commun 510: 416-420, 2019.

24. Zhang $\mathrm{R}$ and Xia T: Long non-coding RNA XIST regulates PDCD 4 expression by interacting with miR-21-5p and inhibits osteosarcoma cell growth and metastasis. Int J Oncol 51: 1460-1470, 2017

25. Stroup DF, Berlin JA, Morton SC, Olkin I, Williamson GD, Rennie D, Moher D, Becker BJ, Sipe TA and Thacker SB: Meta-analysis of observational studies in epidemiology: A proposal for reporting. Meta-analysis of Observational Studies in Epidemiology (MOOSE) group. JAMA 283: 2008-2012, 2000.

26. Enneking WF: A system of staging musculoskeletal neoplasms. Clin Orthop Relat Res 9-24, 1986.

27. Zhang Q, Wang J, Deng F, Yan Z, Xia Y, Wang Z, Ye J, Deng Y, Zhang Z, Qiao M, et al: TqPCR: A touchdown qPCR assay with significantly improved detection sensitivity and amplification efficiency of SYBR green qPCR. PLoS One 10: e0132666, 2015.

28. Livak KJ and Schmittgen TD: Analysis of relative gene expression data using real-time quantitative PCR and the 2(-Delta Delta C(T)) method. Methods 25: 402-408, 2001

29. Higgins JP and Thompson SG: Quantifying heterogeneity in a meta-analysis. Stat Med 21: 1539-1558, 2002.

30. Bowden J, Tierney JF, Copas AJ and Burdett S: Quantifying, displaying and accounting for heterogeneity in the meta-analysis of RCTs using standard and generalised Q statistics. BMC Med Res Methodol 11: 41, 2011.
31. Wells GA, Shea B, O'Connell D, Peterson J, Welch V, Losos M and Tugwell P: The Newcastle-Ottawa Scale (NOS) for assessing the quality of nonrandomized studies in meta-analyses. http://www. ohri.ca/programs/clinical_epidemiology/oxford.asp. Accessed June 15, 2019.

32. Tantai J, Hu D, Yang Y and Geng J: Combined identification of long non-coding RNA XIST and HIF1A-AS1 in serum as an effective screening for non-small cell lung cancer. Int J Clin Exp Pathol 8: 7887-7895, 2015

33. Fang J, Sun CC and Gong C: Long noncoding RNA XIST acts as an oncogene in non-small cell lung cancer by epigenetically repressing KLF2 expression. Biochem Biophys Res Commun 478: 811-817, 2016.

34. Chen DL, Ju HQ, Lu YX, Chen LZ, Zeng ZL, Zhang DS, Luo HY, Wang F, Qiu MZ, Wang DS, et al: Long non-coding RNA XIST regulates gastric cancer progression by acting as a molecular sponge of miR-101 to modulate EZH2 expression. J Exp Clin Cancer Res 35: 142, 2016.

35. Li GL, Wu YX, Li YM and Li J: High expression of long non-coding RNA XIST in osteosarcoma is associated with cell proliferation and poor prognosis. Eur Rev Med Pharmacol Sci 21: 2829-2834, 2017.

36. Chen DL, Chen LZ, Lu YX, Zhang DS, Zeng ZL, Pan ZZ, Huang P, Wang FH, Li YH, Ju HQ and Xu RH: Long noncoding RNA XIST expedites metastasis and modulates epithelial-mesenchymal transition in colorectal cancer. Cell Death Dis 8: e3011, 2017.

37. Wei W, Liu Y, Lu Y, Yang B and Tang L: LncRNA XIST promotes pancreatic cancer proliferation through miR-133a/EGFR. J Cell Biochem 118: 3349-3358, 2017.

38. Du P, Zhao H, Peng R, Liu Q, Yuan J, Peng G and Liao Y: LncRNA-XIST interacts with miR-29c to modulate the chemoresistance of glioma cell to TMZ through DNA mismatch repair pathway. Biosci Rep 37: BSR20170696, 2017.

39. Wang Z, Yuan J, Li L, Yang Y, Xu X and Wang Y: Long non-coding RNA XIST exerts oncogenic functions in human glioma by targeting miR-137. Am J Transl Res 9: 1845-1855, 2017.

40. Mo Y, Lu Y, Wang P, Huang S, He L, Li D, Li F, Huang J, Lin X, Li X, et al: Long non-coding RNA XIST promotes cell growth by regulating miR-139-5p/PDK1/AKT axis in hepatocellular carcinoma. Tumour Biol 39: 1010428317690999, 2017.

41. Sun G, Wang $C$ and Zhang H: Long non-coding RNA XIST promotes cervical cancer cell epithelial-mesenchymal transition through the Wnt/ $\beta$-catenin pathway. Int J Clin Exp Pathol 10: 2333-2339, 2017.

42. Xiong Y, Wang L, Li Y, Chen M, He W and Qi L: The long non-coding RNA XIST interacted with MiR-124 to modulate bladder cancer growth, invasion and migration by targeting androgen receptor (AR). Cell Physiol Biochem 43: 405-418, 2017.

43. Sun W, Zu Y, Fu X and Deng Y: Knockdown of lncRNA-XIST enhances the chemosensitivity of NSCLC cells via suppression of autophagy. Oncol Rep 38: 3347-3354, 2017.

44. Wu X, Dinglin X, Wang X, Luo W, Shen Q, Li Y, Gu L, Zhou Q, Zhu H, Li Y, et al: Long noncoding RNA XIST promotes malignancies of esophageal squamous cell carcinoma via regulation of miR-101/EZH2. Oncotarget 8: 76015-76028, 2017.

45. Kong Q, Zhang S, Liang C, Zhang Y, Kong Q, Chen S, Qin J and Jin Y: LncRNA XIST functions as a molecular sponge of miR-194-5p to regulate MAPK1 expression in hepatocellular carcinoma cell. J Cell Biochem 119: 4458-4468, 2018.

46. Yi SZ, Zhang W, Zhou QY and Cheng GR: 1ncRNA XIST promotes aggressive tumor phenotype and is associated with poor prognosis in esophageal squamous cell carcinoma. Int J Clin Exp Med 11: 8317-8323, 2018.

47. Liang S, Gong X, Zhang G, Huang G, Lu Y and Li Y: The lncRNA XIST interacts with miR-140/miR-124/iASPP axis to promote pancreatic carcinoma growth. Oncotarget 8: 113701-113718, 2017.

48. Sun N, Zhang G and Liu Y: Long non-coding RNA XIST sponges $\mathrm{miR}-34 \mathrm{a}$ to promotes colon cancer progression via Wnt/ $\beta$-catenin signaling pathway. Gene 665: 141-148, 2018.

49. Liu H, Deng H,Zhao Y, Li C and Liang Y: LncRNA XIST/miR-34a axis modulates the cell proliferation and tumor growth of thyroid cancer through MET-PI3K-AKT signaling. J Exp Clin Cancer Res 37: 279, 2018.

50. Hu C, Liu S, Han M, Wang Y and Xu C: Knockdown of lncRNA XIST inhibits retinoblastoma progression by modulating the miR-124/STAT3 axis. Biomed Pharmacother 107: 547-554, 2018. 
51. Zhu H, Zheng T, Yu J, Zhou L and Wang L: LncRNA XIST accelerates cervical cancer progression via upregulating Fus through competitively binding with miR-200a. Biomed Pharmacother 105: 789-797, 2018.

52. Siegel RL, Miller KD and Jemal A: Cancer statistics, 2016. CA Cancer J Clin 66: 7-30, 2016.

53. Yarmishyn AA and Kurochkin IV: Long noncoding RNAs: A potential novel class of cancer biomarkers. Front Genet 6: 145 , 2015.

54. Mercer TR, Dinger ME and Mattick JS: Long non-coding RNAs: Insights into functions. Nat Rev Genet 10: 155-159, 2009.

55. Cui X, Jing X, Long C, Tian J and Zhu J: Long noncoding RNA MEG3, a potential novel biomarker to predict the clinical outcome of cancer patients: A meta-analysis. Oncotarget 8: 19049-19056, 2017.
56. Fan YH, Fang H, Ji CX, Xie H, Xiao B and Zhu XG: Long noncoding RNA CCAT2 can predict metastasis and poor prognosis: A meta-analysis. Clin Chim Acta 466: 120-126, 2017.

57. Zhang S, Chen S, Yang G, Gu F, Li M, Zhong B, Hu J, Hoffman A and Chen M: Long noncoding RNA HOTAIR as an independent prognostic marker in cancer: A meta-analysis. PLoS One 9: e105538, 2014.

58. Zhu J, Kong F, Xing L, Jin Z and Li Z: Prognostic and clinicopathological value of long noncoding RNA XIST in cancer. Clin Chim Acta 479: 43-47, 2018.

(i) (i) $($ This work is licensed under a Creative Commons (c) NG ND Attribution-NonCommercial-NoDerivatives 4.0 International (CC BY-NC-ND 4.0) License. 